\title{
Propagation Modelling in a Container Environment
}

\author{
Emmeric Tanghe, Wout Joseph, Peter Ruckebusch, Luc Martens, and Ingrid Moerman \\ Ghent University/Interdisciplinary institute for BroadBand Technology (IBBT) \\ Department of Information Technology (INTEC), Ghent, Belgium, email: emmeric.tanghe@intec.ugent.be
}

\begin{abstract}
This paper presents propagation modelling in an environment of stacked shipping containers. Three different propagation scenarios are identified for which path loss is determined experimentally.
\end{abstract}

\section{INTRODUCTION}

One of the major tasks of supply chain management is to follow goods, stored in shipping containers, from origin to final destination. This paper presents propagation modelling for a general wireless architecture for container monitoring and tracking, developed within the IBBT MoCo project [1]. The architecture is based around so-called MoCo (for Monitoring of Containers) wireless devices. A MoCo device is attached outside on a container's surface and has three main functionalities: (i) it can wirelessly gather data from sensors inside the container that measure transport parameters such as temperature, humidity, shock, and door status, (ii) it can relay that data to other MoCo devices attached to other containers, and (iii) it can send data to a cloud for storage and retrieval. Obtaining enough wireless link budget for these functionalities can be particularly challenging because of the highly metallic (shielding) nature of the stacked container environment. To assess this, path loss measurements were carried out in this type of environment.

\section{Propagation SCENARIOS}

We distinguish between three types of wireless links between sensors, MoCo devices, and the cloud. Each link type handles one of the MoCo device's main funtionalities (Section I) and is entirely different when it comes to propagation characteristics:

- Intra-container link: a sensor inside the container is communicating with a MoCo device attached outside to that container (IEEE 802.15.4).

- Inter-container link: communication between MoCo devices attached to different containers (IEEE 802.15.4).

- Extra-container link: communication between MoCo devices and the cloud (IEEE 802.11, GSM, UMTS).

In the above list, it is also mentioned which communication standard(s) is (are) suitable for implementing each link.

\section{MeAsurement SETUP AND DATA PROCESSING}

The measurement setup consists of a transmitting and a receiving part (Fig. 1). At the transmitter, a signal generator creates a continuous sine wave which is fed to the transmitting antenna Tx. At the receiving end, a spectrum analyser samples the power at the receiving antenna Rx. As Tx and $\mathrm{Rx}$, vertically polarized half-wave dipole antennas are used.
Following measurements, path loss $P L$ in $\mathrm{dB}$ is calculated as:

$$
P L=P_{T x}-L_{T x}-L_{R x}-P_{R x}
$$

In (1), $P_{T x}$ is the transmit power $(20 \mathrm{dBm}), L_{T x}$ and $L_{R x}$ are the Tx and Rx antenna feeder losses in $\mathrm{dB}$, and $P_{R x}$ is the received power in $\mathrm{dBm}$. It is important to note that the usual calculation of path loss also includes terms in the right-hand side of (1) which exclude the gains of the measurement antennas. This was however not done here: due to the proximity of the metallic container surface, antenna effects cannot be separated from the wireless propagation loss and as such the path loss in (1) includes both phenomena.

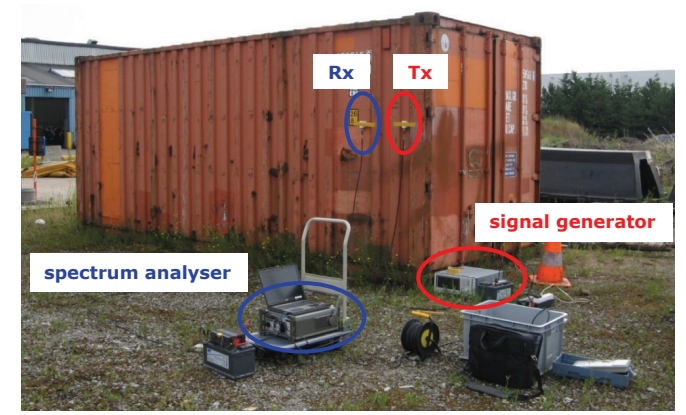

Figure 1. Measurement setup

\section{RESUlTS}

This section discusses path loss results for the intercontainer link (the intra- and extra-container links will be discussed in the full paper). In the MoCo architecture, the inter-container link adopts the IEEE 802.15.4 communication standard (Section II). Path loss is therefore investigated for three frequencies targeted by IEEE 802.15 .4 , namely 433, 868 , and $2400 \mathrm{MHz}$. The measurements are carried out for two common types of container stacking: row and block stacking.

\section{A. Inter-container link for row stacking}

The measurements are carried out along a row of 4 containers (length of $42.7 \mathrm{~m}$ ). The Tx is mounted on the ventilation holes near the top of the first container in the row. The Rx is then moved along the top of the container row at the same height above ground level as the Tx. The Rx is moved in steps of $0.5 \mathrm{~m}$, and at each Rx location, 200 samples of received power $P_{R x}$ are recorded. In total, $83 \mathrm{Rx}$ locations are measured per frequency. Measured path loss is found to agree well to an exponential decay with distance. Path loss $P L$ (in $\mathrm{dB}$ ) is 
fitted to the following model which is function of the distance $d$ (in $\mathrm{m}$ ) between $\mathrm{Tx}$ and $\mathrm{Rx}$ :

$$
P L(d)=a_{0}+a_{1} \cdot d+\chi_{s}+\chi_{t}
$$

In (2), $a_{0}$ and $a_{1}$ are regression parameters, and $\chi_{s}$ and $\chi_{t}$ are zero-mean random variables that account for shadow fading and temporal fading, respectively. $\chi_{s}$ assumes a normal distribution with standard deviation $\sigma_{s}$ while $\chi_{t}$ closely follows a t-location-scale distribution with scale parameter $\sigma_{t}$ and shape parameter $\nu_{t}$. Table I lists estimated values for the parameters of (2), obtained by least-squares fitting.

\begin{tabular}{|cccccc|}
\hline Frequency & $a_{0}[\mathrm{~dB}]$ & $a_{1}[\mathrm{~dB} / \mathrm{m}]$ & $\sigma_{s}[\mathrm{~dB}]$ & $\sigma_{t}[\mathrm{~dB}]$ & $\nu_{t}[-]$ \\
\hline $433 \mathrm{MHz}$ & 60.34 & 0.67 & 6.83 & 0.27 & 1.24 \\
$868 \mathrm{MHz}$ & 56.78 & 0.77 & 5.38 & 0.28 & 1.25 \\
$2400 \mathrm{MHz}$ & 60.9 & 0.55 & 5.48 & 0.39 & 1.20 \\
\hline
\end{tabular}

Table I

INTER-CONTAINER PATH LOSS MODEL PARAMETERS (ROW STACKING)

\section{B. Inter-container link for block stacking}

The measurements were carried out on a 3-D stack of 16 containers ( 4 long, 2 wide, and 2 high). The stack is made up of 20' containers. Fig. 2 shows the container stack schematically: shown are the 8 containers on the ground level and the 8 container on the upper level.

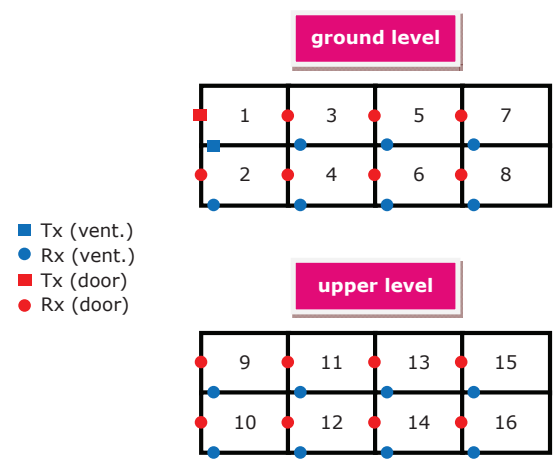

Figure 2. Inter-container scenario for block stacking

Measurements are done for two scenarios. In the first scenario, the MoCo devices are mounted on the containers' ventilation holes (blue symbols). For the second scenario, the MoCo devices are attached to the containers' doors (red symbols). For both scenarios, path loss is measured between the Tx on container 1 (squares) and the Rx's on the other containers (circles). For each Tx-Rx link, 300 samples of received power $P_{R x}$ are recorded.

Fig. 3 shows the path loss results at $433 \mathrm{MHz}$ for each of the numbered containers in Fig. 2. The variability of path loss over the 300 samples is shown as a boxplot. The blue boxplots are for the vent-mounted scenario while the red ones correspond to the door-mounted scenario. For frequencies of 433 and $868 \mathrm{MHz}$, it is found for the majority of containers that the path loss is smaller for the door-mounted scenario than for the vent-mounted scenario. For $2.4 \mathrm{GHz}$ this observation could not be made. A possible qualitative explanation for this is that propagation for the vent-mounted scenario is more difficult, as for this scenario the $\mathrm{Tx}$ is closed in between containers. This means, moreso than for the door-mounted scenario, that propagation paths run mainly in the gaps between containers. This propagation in small gaps is more difficult for the lower frequencies of 433 and $868 \mathrm{MHz}$ compared to $2400 \mathrm{MHz}$ due to the larger wavelength.
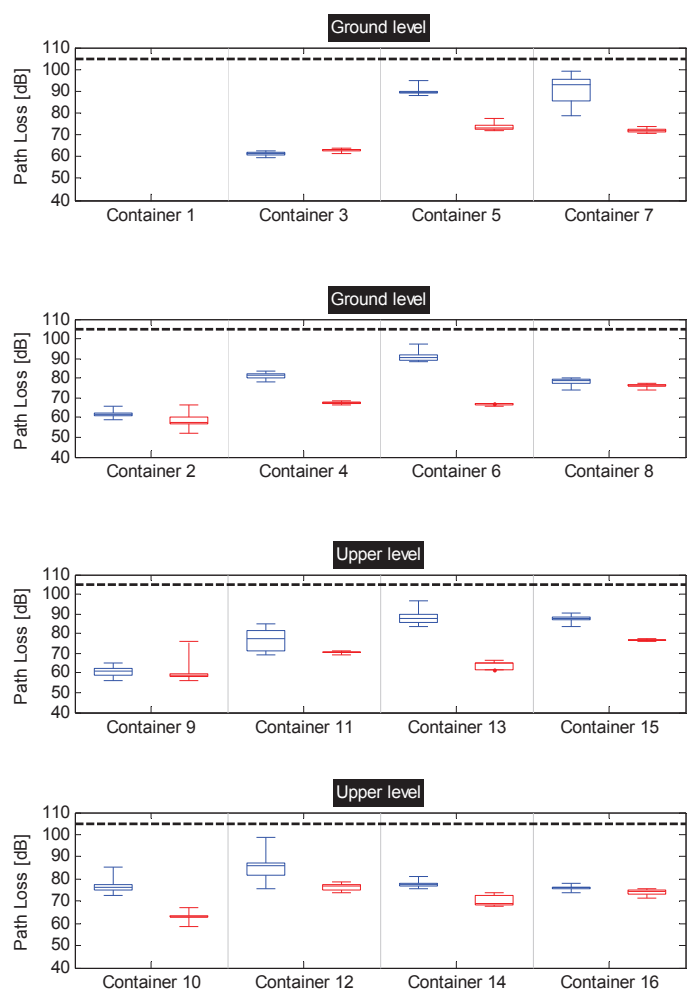

Figure 3. Inter-container path loss boxplots (block stacking, $433 \mathrm{MHz}$ )

\section{CONCLUSIONS}

In this work, path loss modelling in an environment of stacked shipping containers has been presented. Three different propagation scenarios are identified for which path loss is determined experimentally. These propagation scenarios correspond to three different types of wireless links that exist within the MoCo architecture for container monitoring, namely intra-, inter-, and extra-container links. This paper has presented the path loss results for the inter-container link. The intra- and extra-container links will be discussed in the full paper.

\section{ACKNOWLEDGMENT}

This work has been carried out with the financial support of the Interdisciplinary institute for BroadBand Technology (IBBT) project 'Monitoring of Containers (MoCo)'. W. Joseph is a Postdoctoral Fellow of the FWO-V (Research FoundationFlanders).

\section{REFERENCES}

[1] Online: http://www.ibbt.be/en/projects/overview-projects/p/detail/moco-2 